\title{
Potencial de utilização de resíduos da cultura de soja tratados com água e hidróxido de sódio para produção de painéis aglomerados
}

\author{
Potential for use of waste from soybean culture treated with water and sodium hydroxide for \\ production of agglomerated panels
}

Potencial de aprovechamiento de residuos del cultivo de soja tratados con agua e hidróxido de sodio para la producción de paneles aglomerados

\section{Resumo}

O Brasil é um dos maiores produtores de soja e, consequentemente, gera uma grande quantidade de resíduos provenientes dessa cultura, os quais poderiam ser aproveitados na confecção de aglomerados. Este trabalho objetivou analisar as características físico-mecânicas de painéis aglomerados, produzidos com partículas de resíduos de soja e eucalipto, tratadas em água fria e hidróxido de sódio. Adotou-se uma proporção de 50:50 de partículas de eucalipto e casquilho de soja. A densidade nominal dos painéis foi $0,60 \mathrm{~g} / \mathrm{cm}^{3}$. Foi utilizado adesivo uréia-formaldeído com teor de sólidos de $12 \%$. Foram adotados três tratamentos: partículas tratadas com água fria e partículas tratadas com $\mathrm{NaOH}$ (com concentração de $1 \%$, além de um tratamento com partículas controle. Os componentes químicos dos materiais lignocelulósicos foram quantificados para as três situações. Os parâmetros do ciclo de prensagem foram: 4 $\mathrm{MPa}$ (pressão), $150{ }^{\circ} \mathrm{C}$ (temperatura), durante $8 \mathrm{~min}$. Foram observadas reduções nos teores de extrativos totais, após os tratamentos. Em relação às propriedades físicas, os tratamentos aplicados nas partículas não demonstraram eficiência, pois não trouxeram melhorias para a estabilidade dimensional dos painéis e nenhum dos tratamentos atingiram ao valor mínimo especificado pela norma utilizada. Para as propriedades mecânicas, os painéis produzidos com partículas tratadas com água atenderam as exigências para o MOE. Para as propriedades de tração perpendicular e dureza Janka, todos os painéis alcançaram os valores especificados pela NBR 14810 (2013). As outras propriedades testadas (MOR, APS e APT) não atenderam a norma. Os tratamentos aplicados nas partículas de soja e eucalipto não demonstraram eficiência para as propriedades físicas, porém algumas propriedades mecânicas atenderam aos requisitos mínimos da NBR 14810 (2013) para painéis não estruturais para uso interno em ambiente seco (Tipo P2). Palavras-chave: Água fria; Hidróxido de sódio; Casquilhos de soja; Análise química; Mistura de partículas.

\section{Abstract}

Brazil is one of the largest soybean producers and, consequently, generates a large amount of residues from this culture, which could be used in the manufacture of agglomerates. This work aimed to analyze the physico-mechanical 
characteristics of agglomerated panels, produced with particles of soy and eucalyptus residues, treated in cold water and sodium hydroxide. A 50:50 ratio of eucalyptus particles and soybean bushes was adopted. The nominal density of the panels was $0.60 \mathrm{~g} / \mathrm{cm}^{3}$. Urea-formaldehyde adhesive with a solids content of $12 \%$ was used. Three treatments were adopted: particles treated with cold water and particles treated with $\mathrm{NaOH}$ (with a concentration of $1 \%$, in addition to a treatment with control particles. The chemical components of the lignocellulosic materials were quantified for the three situations. The parameters of the pressing cycle were : $4 \mathrm{MPa}$ (pressure), $150{ }^{\circ} \mathrm{C}$ (temperature), for $8 \mathrm{~min}$. Reductions were observed in the contents of total extractives, after the treatments. Regarding the physical properties, the treatments applied to the particles did not demonstrate efficiency, as they did not bring improvements for the dimensional stability of the panels and none of the treatments reached the minimum value specified by the standard used. For the mechanical properties, the panels produced with water-treated particles met the requirements for the MOE. For the perpendicular tensile properties and Janka hardness, all the panels reached the values specified by NBR 14810 (2013). The other properties tested the (MOR, APS and APT) did not meet the standard. The treatments applied to soybean and eucalyptus particles did not demonstrate efficiency for physical properties, but some mechanical properties met the minimum requirements of NBR 14810 (2013) for non-structural panels for indoor use in a dry environment (Type P2).

Keywords: Cold water; Sodium hydroxide; Soy bushes; Chemical analysis; Particulate mixing.

\section{Resumen}

Brasil es uno de los mayores productores de soja y, en consecuencia, genera una gran cantidad de residuos de este cultivo, que podrían ser utilizados en la fabricación de aglomerados. Este trabajo tuvo como objetivo analizar las características físico-mecánicas de paneles aglomerados, producidos con partículas de residuos de soya y eucalipto, tratados en agua fría e hidróxido de sodio. Se adoptó una relación 50:50 de partículas de eucalipto y arbustos de soja. La densidad nominal de los paneles fue de $0,60 \mathrm{~g} / \mathrm{cm}^{3}$. Se utilizó adhesivo de urea-formaldehído con un contenido de sólidos del $12 \%$. Se adoptaron tres tratamientos: partículas tratadas con agua fría y partículas tratadas con $\mathrm{NaOH}$ (con una concentración del $1 \%$, además de un tratamiento con partículas control. Se cuantificaron los componentes químicos de los materiales lignocelulósicos para las tres situaciones. Los parámetros del ciclo de prensado fueron: 4 $\mathrm{MPa}$ (presión), $150{ }^{\circ} \mathrm{C}$ (temperatura), durante $8 \mathrm{~min} \mathrm{Se}$ observaron reducciones en los contenidos de extractivos totales, después de los tratamientos En cuanto a las propiedades físicas, los tratamientos aplicados a las partículas no demostraron eficiencia, ya que no trajeron mejoras para la estabilidad dimensional de los paneles y ninguno de los tratamientos alcanzó el valor mínimo especificado por la norma utilizada.Para las propiedades mecánicas, los paneles producidos con partículas tratadas con agua cumplieron con los requisitos del MOE. las propiedades de tracción perpendicular y dureza Janka, todos los paneles alcanzaron los valores especificados por la NBR 14810 (2013). el (MOR, APS y APT) no cumplía con el estándar. Los tratamientos aplicados a las partículas de soja y eucalipto no demostraron eficiencia para las propiedades físicas, pero algunas propiedades mecánicas cumplieron con los requisitos mínimos de la NBR 14810 (2013) para paneles no estructurales para uso interior en ambiente seco (Tipo P2).

Palabras clave: Agua fría; Hidróxido de sódio; Arbustos de soja; Análisis químico; Mezcla de partículas.

\section{Introdução}

No Brasil, a madeira proveniente de florestas plantadas, principalmente de Pinus spp e Eucalyptus spp, são fontes importantes de matérias-primas para a fabricação do aglomerado. A área plantada em 2020 no país, por exemplo, passou para 9,55 milhões de hectares para cultivos industriais (Instituto Brasileiro de Atuária - IBÁ, 2021).

Dentre as espécies mais plantadas destaca-se o Eucalyptus grandis, cultivado em grandes proporções no Brasil e em outros países da América Central e do Sul (Empresa Brasileira de Pesquisa Agropecuária - EMPRAPA, 2019). Por outro lado, a demanda por produtos à base de madeira vem aumentando num ritmo cada vez maior, por isso além da madeira de eucalipto, que já abastece as indústrias é interessante verificar outras fontes de materiais lignocelulósicos, que possam substituir a madeira de forma total ou parcial e assim desenvolver novos produtos.

Experimentos destinados à avaliação do potencial tecnológico de resíduos (florestais, agrícolas e agroindustriais) são importantes do ponto de vista científico, no sentido de contribuir com novas opções de matéria prima para as indústrias do setor madeireiro. Com a utilização dos resíduos, agrega-se valor aos mesmos, além de atenuar os descartes no meio ambiente. Pesquisadores vêm mostrando a viabilidade do emprego de resíduos agroindustriais, associados ou não a madeira, que possuem uso potencial, tais como: palha de milho, resíduo de sorgo, resíduos de coco de babaçu, espiga de milho, casca de arroz, bambu e bagaço de cana de açúcar, dentre outros (Silva et al., 2015; Guimarães Júnior et al., 2016, Machado et al., 
2017, Scatolino et al., 2017; César et al., 2017, Bazzetto et al., 2019, Brito \& Bortoletto júnior, 2019, 2020).

Uma alternativa interessante seria utilizar resíduos de soja para produção de painéis aglomerados, visto que, o Brasil ocupa a segunda posição em produção mundial de soja em grãos. Segundo o levantamento realizado a produção recorde de soja está estimada em 120,9 milhões de toneladas, para a safra de 2019/2020, ganho de 5,1\% em relação à safra passada (Companhia Nacional de Abastecimento - CONAB, 2020). Essa produção, consequentemente gera grandes volumes de resíduos, estima-se que a cultura da soja produza cerca de 2.700 t de resíduos para cada 1 mil t de grãos processados (Matos, 2005).

Geralmente, tais resíduos são direcionados para fabricar ração para bovinos; porém, os mesmos possuem propriedades químicas que os credenciam como possíveis matérias-primas para serem utilizados em outros segmentos industriais, como na produção de painéis de partículas (Martins et al., 2018). Destacam-se dois experimentos já realizados com resíduos de soja, como matéria prima para fabricação de painéis de partículas (Martins et al., 2018, Guimarães et al., 2019).

Durante a condução do experimento, Guimarães et al. (2019) notaram que os resíduos de soja possuem alto teor de extrativos e teores elevados destes componentes podem dificultar o processo de colagem das partículas, além de afetar a qualidade do painel. Por essa razão a submissão das partículas de resíduo de soja a tratamentos adequados e eficientes, visando remover constituintes químicos indesejados, para utilizá-las na manufatura de painéis de partículas aglomeradas, se mostra como uma boa opção (Guimarães et al. 2014). Algumas pesquisas, neste sentido, já foram realizadas com o pré-tratamento de partículas de materiais lignocelulósicos e seus efeitos sobre as propriedades tecnológicas de painéis aglomerados, tais como: Zhang e Hu, (2014); Guimarães et al., (2014); Jumhuri et al, (2014), Cao et al., (2017) e Brito e Bortoletto Júnior, (2020)5, que utilizaram tratamentos com solução de hidróxido de sódio e água.

Assim, o objetivo deste trabalho foi analisar as características físico-mecânicas de painéis aglomerados, produzidos com partículas de resíduos de soja e eucalipto, tratadas em água fria e hidróxido de sódio.

\section{Metodologia}

\subsection{Partículas}

Os resíduos de soja (parte aérea e casquilho) utilizados como matéria prima para produção dos painéis estavam disponíveis no Departamento de Agricultura da Universidade Federal de Lavras (UFLA), onde o experimento foi realizado. Foram utilizadas árvores de Eucalyptus grandis com idade de 10 anos, provenientes de plantios experimentais do campus da própria universidade.

Os resíduos de soja e a madeira de eucalipto foram transformados em partículas com o auxílio de um moinho de martelo. Em seguida foi feita a classificação dos materiais em um conjunto de peneiras e a fração de partículas com morfologia mais adequada para a produção de painéis foi constituída com partículas de 16 mesh. Posteriormente, as partículas de soja e eucalipto foram secas em estufa até atingirem $3 \%$ de umidade e reservadas.

\subsection{Tratamento das partículas}

As partículas, não tratadas e secas em estufa $(103 \pm 2){ }^{\circ} \mathrm{C}$, com teor de $3 \%$ de umidade, foram utilizadas para produção dos painéis do grupo controle. O tratamento das partículas em água fria consistiu em submergi-las em água a temperatura ambiente por 24 horas. Posteriormente, as fibras foram secas ao ar livre por 48 horas e em estufa até atingirem o teor de $3 \%$ de umidade. Já o tratamento com $\mathrm{NaOH}$ consistiu na imersão do material com concentração de 1,0 \%. As partículas ficaram em contato com a solução alcalina por 2 horas. Após esse período, procedeu-se a lavagem das partículas em água corrente para evitar a deposição de algum componente químico na superfície. Em seguida, as fibras foram secas ao ar 
livre por 48 horas e depois a secagem foi complementada em estufa. A Figura 1 representa as partículas de casquilho de soja e eucalipto utilizadas antes e após os tratamentos.

Figura 1. Partículas antes e após o tratamento: soja (a) e eucalipto (b).

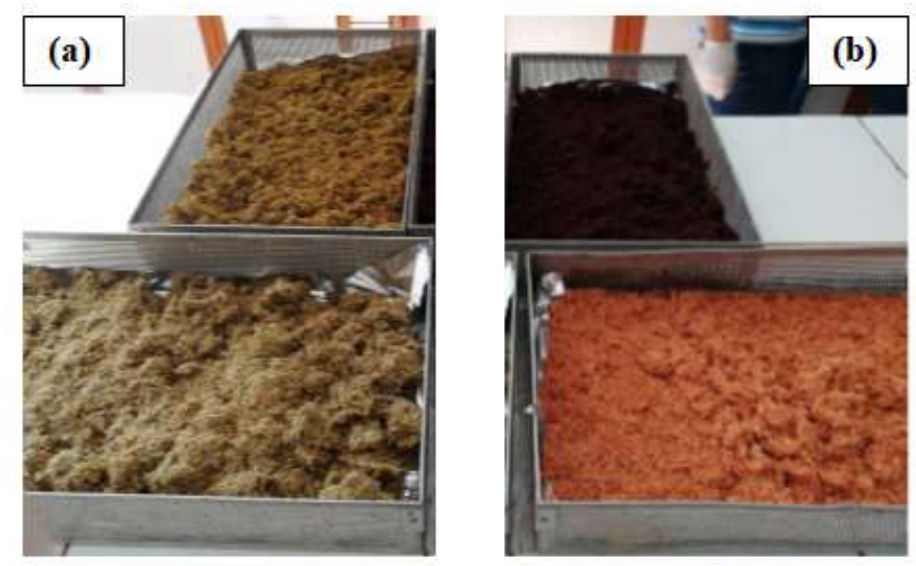

Fonte: Autores (2021).

\subsection{Análise química das partículas}

Uma porção adequada de partículas de casquilho e de soja foram selecionadas para determinar a análise química. O material foi transformado em serragem utilizando um moinho de martelo e, posteriormente, foi classificado em peneiras e as partículas selecionadas foram aquelas que ficaram retidas em peneira de 60 mesh. Os constituintes químicos foram analisados por meio de triplicatas. As partículas foram caracterizadas em relação aos seguintes componentes químicos: extrativos totais NBR 14853 (ABNT, 2010) e lignina insolúvel - NBR 7989 (ABNT, 2010). O teor de holocelulose foi calculado por simples diferença.

\subsection{Produção dos painéis de partículas}

A densidade nominal dos painéis foi $0,60 \mathrm{~g} / \mathrm{cm}^{3}$. Foi utilizado adesivo a base de Ureia-Formaldeído (UF) com teor de sólidos de $12 \%$, em relação à massa seca das partículas. Conforme a ficha técnica, que acompanha o adesivo, as características físico-químicas foram as seguintes: teor de sólidos de $64,60 \%$, viscosidade de $480 \mathrm{cP}$, gel time de $51 \mathrm{~s} \mathrm{e} \mathrm{pH} \mathrm{de} \mathrm{8,55.} \mathrm{A}$ proporção adotada foi de $50 \%$ de partículas de eucalipto e $50 \%$ de partículas de casquilho de soja. Utilizou-se uma encoladeira, para aspersão homogênea do adesivo sobre as partículas. Após este processo, as partículas foram dispostas de forma manual dentro de uma caixa de madeira, com dimensões de 25 x 25 x 1,5 cm (largura, comprimento e espessura), para formação do colchão de partículas.

Posteriormente foi realizada a pré-prensagem, com pressão de 0,4 $\mathrm{MPa}$, em uma prensa hidráulica e à temperatura ambiente. Os painéis foram então transportados até uma prensa automatizada com capacidade de 40 toneladas e foram adotados os seguintes parâmetros de fabricação: temperatura de $150{ }^{\circ} \mathrm{C}$ e pressão de $4 \mathrm{MPa}$ por 8 minutos. Durante o processo foram utilizados espaçadores de metal com espessura de 15,00 $\mathrm{mm}$. Em seguida, os painéis foram acondicionados em sala climatizada ( $20 \pm 2{ }^{\circ} \mathrm{C}$ e $65 \pm 3 \%$ UR) até estabilização da massa, para posterior retirada das extremidades, com o auxílio de uma serra circular e produção dos corpos de prova para os ensaios, conforme designado pela NBR 14810 (ABNT, 2013),

Os ensaios físicos realizados foram: densidade aparente (DA), absorção de água após 2 h (AA 2 h) e 24 h (AA 24 h) de imersão, inchamento em espessura após 2 h (IE 2 h) e 24 h (IE 24 h) de imersão. O desempenho mecânico foi avaliado por meio das seguintes propriedades: módulo de elasticidade (MOE) e módulo de ruptura (MOR) a flexão estática, dureza Janka (DJ), resistência à tração perpendicular (TP), resistência ao arrancamento do parafuso na superfície (RAPS) e topo (RAPT) 
(NBR 14810-3 (ABNT, 2006)).

\subsection{Análise dos dados}

Foram produzidos três painéis para cada tratamento totalizando nove unidades experimentais. A análise química foi realizada de forma descritiva para os parâmetros em questão (extrativos, lignina e holocelulose). Para verificar os efeitos dos tratamentos na qualidade dos painéis, foi adotado um delineamento inteiramente casualizado para avaliar o fator tratamento, em três níveis: controle, água fria e $\mathrm{NaOH}$. Os dados das propriedades analisadas foram submetidos à análise de variância (ANOVA) e foi utilizado o teste de Scott-Knott a $5 \%$ de probabilidade, para comparação das médias.

Adicionalmente, os valores médios das propriedades físicas e mecânicas dos painéis de todos os tratamentos adotados foram confrontados com os parâmetros requeridos pela NBR 14810 (ABNT, 2006) e NBR 14810 (ABNT, 2013), que estipulam os requisitos para painéis não estruturais para uso interno em condições secas (Tipo P2).

\section{Resultados e Discussão}

\subsection{Caracterização dos materiais lignocelulósicos}

Na tabela 1 estão descritos os valores médios da análise química da madeira de eucalipto e resíduo de soja.

Tabela 1. Propriedades dos materiais lignocelulósicos.

\begin{tabular}{ccccc}
\hline Material & Tratamentos & Extrativos $(\%)$ & Lignina (\%) & Holocelulose (\%) \\
\hline \multirow{3}{*}{ Eucalyptus grandis } & Controle & $6,61^{(2,49)}$ & $16,75^{(1,36)}$ & $76,64^{(1,13)}$ \\
\cline { 2 - 4 } & NaOH & $4,64^{(0,23)}$ & $21,97^{(2,20)}$ & $73,39^{(2,43)}$ \\
\cline { 2 - 4 } & Água Fria & $3,36^{(1,98)}$ & $22,25^{(2,45)}$ & $74,39^{(4,43)}$ \\
\hline \multirow{2}{*}{ Resíduos de soja } & Controle & $13,69^{(0,14)}$ & $22,04^{(0,47)}$ & $64,27^{(0,61)}$ \\
\cline { 2 - 4 } & NaOH & $4,93^{(3,27)}$ & $23,45^{(0,11)}$ & $71,62^{(3,38)}$ \\
\cline { 2 - 4 } & Água Fria & $5,00^{(1,09)}$ & $20,62^{(3,05)}$ & $74,38^{(1,96)}$ \\
\hline
\end{tabular}

*Nota: Valores entre parênteses referem-se aos desvios-padrões das médias. Fonte: Autores (2021).

Conforme os dados descritos na Tabela 1, é possível observar que as partículas controle de resíduo de soja evidenciaram teor de extrativos superior ao dobro do teor obtido para as partículas controle de eucalipto. Este fato também observado por Martins et al. (2018) ao estudarem os teores de extrativos no resíduo de soja e Eucalyptus grandis. Os autores notaram que o teor de extrativo quantificado na soja $(26,72 \%)$ foi cerca de oito vezes maior, em relação aos teores de extrativos obtidos para a madeira.

Os tratamentos realizados (água fria e hidróxido de sódio) demonstraram eficiência para remoção dos extrativos nas espécies avaliadas. Para as partículas de eucalipto tratadas em água, observou-se uma redução correspondente a 49,17 \% no teor de extrativos. Para as partículas tratadas com $\mathrm{NaOH}$ a redução foi equivalente a 29,81 \%. Provavelmente as partículas tratadas em água fria perderam compostos secundários, como amido e açúcar. Os teores de extrativos das partículas de soja tratadas com água foram reduzidos em $63,48 \%$, enquanto o teor de redução das partículas tratadas com NaOH foi 64,00 \%. Os tratamentos demonstraram maior eficiência para remoção dos extrativos das partículas de resíduo de soja. De forma geral, o tratamento realizado com água fria demonstrou maior remoção de extrativos para ambas as partículas.

Valores elevados de extrativos em materiais lignocelulósicos estão diretamente relacionados com a redução da permeabilidade e capacidade higroscópica da matéria prima; além disso, os extrativos podem causar estouros no final da fase de prensagem, além de interferir na cura do adesivo, afetando a qualidade de colagem entre as partículas (Iwakiri, 2005; Surdi et al., 2019). Martins et al. (2018) obtiveram valor médio de 26,72\%, para o teor de extrativos totais do resíduo de soja, valor 
quase duas vezes maior do que do teor obtido no presente estudo. A faixa recomendável para o teor de extrativos presentes em matérias primas para produção de painéis é de 4 a $10 \%$ e, por isso, é interessante aplicar tratamentos nas partículas (Iwakiri \& Trianoski, 2020; Klock \& Andrade, 2013).

Em relação à madeira de eucalipto, Zanuncio et al. (2014), realizaram a composição química da madeira de Eucalyptus grandis e obtiveram teor de extrativos totais correspondente a $6,05 \%$, valor semelhante ao obtido no presente estudo. Sabe-se que os teores de extrativos podem variar conforme a idade, metodologias de extração, solvente, condições edafoclimáticas e técnicas de manejo.

Em relação à lignina observou-se que as partículas de eucalipto tratadas obtiveram um aumento aparente no seu teor, assim como os resíduos de soja tratados com hidróxido que também aparentaram um incremento. Esperava-se uma redução no teor de lignina, pois o $\mathrm{NaOH}$, por exemplo, remove certas quantidades deste componente, ceras e óleos da parede celular; entretanto como o teste de médias não foi realizado, não é possível inferir sobre os resultados com maior precisão (Moraes, 2010).

O valor encontrado para o teor de lignina do casquilho de soja está situado entre os valores descritos na literatura para outros resíduos. Machado et al. (2017) trabalharam com resíduos de epicarpo e endocarpo de coco babaçu e obtiveram para o epicarpo $35,70 \%$ e para o endocarpo encontraram 35,35\%. Scatolino et al. (2017) realizaram um estudo com pergaminho de café e relataram teor médio de $28,32 \%$. Brito et al. (2021) reportaram para a casca de pistache valor médio de $24,74 \%$.

Para a madeira de eucalipto, os valores obtidos na literatura para o teor de lignina foram superiores aos encontrados nesta pesquisa. Sette Junior et al. (2014) encontraram teor médio de 27,80 \% e Garcia et al. (2021) obtiveram 30,11\%. A lignina tem uma estrutura complexa e irregular que confere ao material alta rigidez. Por esta razão, espera-se que painéis produzidos com maiores teores de lignina apresentem maior hidrofobicidade à água e maior resistência mecânica (Sweet \& Winandy, 1999; Khedari et al., 2004).

É possível observar na Tabela 1 reduções no teor de holocelulose para as partículas de madeira tratadas em água fria e hidróxido de sódio, em relação às partículas controle. Em parte, isso pode ser explicado, em função das reações que ocorreram durante os tratamentos. Dentre as substâncias estruturais de madeira (celulose, hemiceluloses e lignina), as hemiceluloses são as menos resistentes à ação dos álcalis. Estes compostos se dissolvem em álcalis e essa propriedade depende de sua composição e estrutura. Os fatores que exercem o papel mais importante nesse processo incluem: comprimento das cadeias macromoleculares, grau de ramificação, organização espacial, presença de grupos carboxila, bem como distribuição nas paredes celulares entre seus outros constituintes (Kin, 1980). Supõe-se que, no caso das partículas tratadas com $\mathrm{NaOH}$, tenha sido eliminada uma porcentagem do material lignocelulósico, visto que a concentração da solução foi $1 \%$, durante 2 horas e, consequentemente, essa perda do material pode ter sido contabilizada como um aumento aparente no teor da lignina.

Uma tendência contrária foi observada para as partículas de casquilho de soja que evidenciaram um aumento no teor de holocelulose para ambos os tratamentos aplicados. O valor encontrado para o teor de holocelulose do casquilho de soja foi superior a outros valores relatados na literatura. Machado et al. (2017) obtiveram teores médios de 53,13\% para o epicarpo e $55,52 \%$ para o endocarpo. Scatolino et al. (2017) obtiveram para a fração de holocelulose e cinzas teor médio de 45,43\% e Brito et al. (2021) obtiveram para cascas de pistache teor médio de 53,31\%, para a mesma fração.

Em relação à madeira de eucalipto o teor ficou entre os valores descritos na literatura. Segundo Sette Junior et al. (2014) o teor médio obtido foi de 69,80\%, enquanto Garcia et al. (2021), encontraram 60,29\%.

Pode-se afirmar que existe uma semelhança entre os valores dos componentes químicos do casquilho e do eucalipto (uma das principais matérias primas utilizadas para produção de painéis aglomerados), exceto para o teor de extrativos, por 
isso é necessário o tratamento das partículas. Portanto, houve influência dos tratamentos nos componentes químicos, que podem influenciar as propriedades físico-mecânicas dos painéis.

\subsection{Propriedades físicas dos painéis}

A Tabela 2 representa o comportamento dos painéis em relação às propriedades físicas.

Tabela 2. Valores médios das propriedades físicas dos painéis aglomerados.

\begin{tabular}{cccccc}
\hline Tratamentos & $\begin{array}{c}\text { DA } \\
\left(\mathrm{g} / \mathrm{cm}^{3}\right)\end{array}$ & $\begin{array}{c}\text { AA } 2 \mathrm{~h} \\
(\%)\end{array}$ & $\begin{array}{c}\text { AA } 24 \mathrm{~h} \\
(\%)\end{array}$ & $\begin{array}{c}\text { IE } 2 \mathrm{~h} \\
(\%)\end{array}$ & $\begin{array}{c}\text { IE } 24 \mathrm{~h} \\
(\%)\end{array}$ \\
\hline Controle & $0,55^{(0,02)} \mathrm{a}$ & $111,75^{(2,54)} \mathrm{a}$ & $139,13^{(6,20)} \mathrm{a}$ & $43,72^{(6,77)} \mathrm{a}$ & $51,39^{(11,58)} \mathrm{a}$ \\
Água & $0,56^{(0,03)} \mathrm{a}$ & $110,18^{(11,77)} \mathrm{a}$ & $136,59^{(16,82)} \mathrm{a}$ & $82,73^{(25,27)} \mathrm{b}$ & $53,25^{(13,28)} \mathrm{a}$ \\
$\mathrm{NaOH}$ & $0,54^{(0,01)} \mathrm{a}$ & $228,77^{(5,60)} \mathrm{b}$ & $240,74^{(2,78)} \mathrm{b}$ & $\left.80,06^{(0,81}\right) \mathrm{b}$ & $98,27^{(3,03)} \mathrm{b}$ \\
\hline
\end{tabular}

*Nota: Médias seguidas de letras iguais não diferem entre si segundo o teste de Scott-Knott $(\boldsymbol{\alpha}=0,05)$. Valores entre parênteses representam o desvio padrão. Fonte: Autores (2021).

Conforme os dados descritos na Tabela 2, observa-se que a massa específica nominal planejada foi diferente dos valores médios da densidade aparente do painel. Esse fato é comum em laboratório e pode ser explicado pelas perdas de insumos utilizados na produção dos painéis durante a formação do colchão. Outra causa seria o retorno em espessura dos painéis, após a retirada da prensa quente e acondicionamento, com consequente aumento do volume e redução da densidade nominal inicial dos painéis (Guimarães et al., 2016). Alguns pesquisadores já relataram essa ocorrência (Guimarães et al., 2016; Bazzetto et al., 2019; Brito et al., 2020 e Brito et al., 2021).

Os painéis de todos os tratamentos podem ser considerados de média densidade, uma vez que a NBR 14810 (ABNT, 2013) classifica dessa forma as chapas com valores entre 0,55 e $0,75 \mathrm{~g} / \mathrm{cm}^{3}$. É importante classificar a densidade dos painéis devido a influência dos valores mínimos de inchamento em espessura, absorção de água, módulo de elasticidade, módulo de ruptura e tração perpendicular (Machado et al., 2017).

Observa-se para as propriedades físicas um comportamento semelhante para AA e IE, no qual os valores obtidos refletem a baixa estabilidade dimensional. As partículas de madeira e resíduos lignocelulósicos, quando expostos à umidade podem sofrer alterações e, em alguns casos, a magnitude desse fenômeno é um fator limitante na utilização da matéria prima (Machado et al., 2017).

Em relação à taxa de absorção de água, observou-se o mesmo comportamento em ambos os tempos de imersão (AA $2 \mathrm{~h}$ e AA 24h). Nota-se diferença significativa entre os tratamentos e os painéis constituídos com partículas tratadas com hidróxido de sódio $(\mathrm{NaOH})$, evidenciaram as maiores taxas de absorção. Provavelmente os extrativos retirados dos lumes celulares, por meio do tratamento aplicado nas partículas, contribuíram com maiores espaços vazios nos painéis e facilitaram a entrada de água uma vez que a absorção é um processo físico e atua por capilaridade.

Para o IE $2 \mathrm{~h}$ observou-se que os painéis produzidos com partículas tratadas em água quente e hidróxido de sódio foram equivalentes estatisticamente, diferindo daqueles produzidos com partículas controle que evidenciaram o menor inchamento. Em relação ao IE $24 \mathrm{~h}$ observou-se que os painéis constituídos com partículas tratadas em $\mathrm{NaOH}$ diferiram estatisticamente dos painéis dos tratamentos T1 e T2, evidenciando maior valor médio e, portanto, baixa estabilidade dimensional.

Comportamento semelhante foi obtido por Zheng et al. (2005) ao trabalharam com painéis aglomerados constituídos de partículas de Agropyron elongatum. Foram utilizados dois adesivos: difenilmetano diisocianato (PMDI) com $4 \%$ de teor de sólidos (ciclo de prensagem: $2 \mathrm{MPa}$ e $140{ }^{\circ} \mathrm{C}$ por $8 \mathrm{~min}$ ) e UF, com $7 \%$ de teor de sólidos (ciclo de prensagem: $2 \mathrm{MPa}$ e 160 
${ }^{\circ} \mathrm{C}$ por $4 \mathrm{~min}$ ). Os autores não utilizaram parafina. A densidade selecionada foi $0,73 \mathrm{~g} / \mathrm{cm}^{3}$. Foram avaliados os efeitos dos adesivos e um tratamento com $\mathrm{NaOH}$ em concentração de 1\%. Para os painéis produzidos com UF, os pesquisadores notaram que a absorção de água e o inchamento em espessura, para 2 e 24 horas, aumentaram cerca de $200 \%$ em comparação com o painel de partículas não tratadas. Os autores destacam que esse resultado pode ter ocorrido por vários motivos: o $\mathrm{NaOH}$ pode ter reagido com alguns componentes da matéria prima e alterado a superfície e/ou a estrutura interna, que impediu que os adesivos se ligassem efetivamente às partículas. $\mathrm{O}$ tratamento com $\mathrm{NaOH}$ pode ter destruído a capacidade da matéria prima reter água durante a prensagem a quente e reduzido a afinidade entre o adesivo (PMDI) e a matéria prima utilizada para fabricação do painel. Isso poderia impedir o adesivo (PMDI) de formar poliureias reticuladas com água na matéria prima e reduzir as ligações químicas através de ligações de hidrogênio e ligações covalentes de poliuretano com a matéria prima utilizada.

Guimarães et al. (2014) trabalharam com painéis constituídos com partículas de pseudocaule de bananeira. Os pesquisadores adotaram cinco tratamentos e dentre eles o $\mathrm{NaOH}$. Foram produzidos painéis com densidade média $\left(0,70 \mathrm{~g} / \mathrm{cm}^{3}\right)$, colados com $12 \%$ (em relação à massa de partículas) do adesivo ureia-formaldeído. O ciclo de prensagem foi realizado a $160^{\circ} \mathrm{C}$ e à $4 \mathrm{MPa}$ por $8 \mathrm{~min}$. Durante o experimento, os pesquisadores observaram que os painéis constituídos com partículas tratadas com $\mathrm{NaOH}$ evidenciaram as maiores taxas de absorção de água e inchamento em ambos os tempos de imersão. Os autores afirmaram que a baixa resistência à absorção de água encontrada para painéis constituídos com partículas tratadas com $\mathrm{NaOH}$ pode ser parcialmente atribuída ao menor teor de lignina da matéria-prima utilizada, por ser um componente hidrofóbico.

Martins et al. (2018) utilizaram madeira do híbrido Eucalyptus urophylla e Eucalyptus grandis em composição com a vagem de soja, nas proporções de $0,25,50,75$ e $10 \%$. A densidade nominal dos painéis foi $0,50 \mathrm{~g} / \mathrm{cm}^{3}$ e espessura nominal de $15,70 \mathrm{~mm}$. A resina ureia-formaldeído foi utilizada em uma quantidade de $12 \%$ em relação a massa de partículas e não foi utilizada emulsão de parafina. Foi realizada uma pré-prensagem em temperatura ambiente com pressão equivalente a 0,4 MPa e posteriormente foi feita a prensagem a quente $\left(8\right.$ minutos a $180{ }^{\circ} \mathrm{C}$ e a uma pressão de $\left.3,92 \mathrm{MPa}\right)$. Os autores obtiveram para os painéis fabricados com $50 \%$ de partículas de eucalipto e $50 \%$ de partículas de resíduo de soja, valores médios para AA 2h, AA 24h, IE $2 \mathrm{~h}$ e IE 24h, correspondentes a 120,15; 125,81; 22,59 e 31,54\%. Com exceção do valor obtido para AA 2h, todos os outros valores citados foram superiores aos obtidos no presente estudo.

Guimarães et al. (2019) avaliaram o efeito da substituição da madeira por casquilho de soja nas propriedades físicas e mecânicas de painéis. Os painéis foram produzidos com densidade nominal de $0,60 \mathrm{~g} / \mathrm{cm}^{3}$. Foram adotadas cinco porcentagens de mistura de partículas: $0,25,50,75$ e $100 \%$ de casca de soja e eucalipto. Utilizou-se teor de adesivo correspondente a $12 \%$ a base de ureia-formaldeído. Os parâmetros do ciclo de prensagem foram: pressão de 4,0 $\mathrm{MPa}$ e temperatura de $180{ }^{\circ} \mathrm{C}$ durante 15 minutos. Martins et al. (2018) e Guimarães et al. (2019), observaram que os painéis produzidos com resíduos de soja evidenciaram aumento de suas propriedades físicas e redução das mecânicas, na medida em que as partículas de madeira foram substituídas pelas partículas de resíduo.

Guimarães et al. (2019) obtiveram para as propriedades de absorção de água em 2 horas valores médios equivalentes a 24,9 e 118,59 \%; para 24 horas de imersão, os valores foram situados entre 49,62 e 140,10 \%. Em relação ao inchamento em espessura (IE), os valores obtidos variaram entre 10,00 a 75,95 \% e 17,49 a 80,49 \%, para 2 e 24 de imersão, respectivamente. Sendo assim, os valores obtidos na presente pesquisa oscilaram entre os valores citados.

$\mathrm{O}$ tratamento químico com $\mathrm{NaOH}$ também pode causar inchamento na estrutura cristalina da celulose, podendo facilitar a entrada de água (Sellers et al., 1988; Joseleau et al., 2004; Khedari et al., 2004; John \& Anandjiwala, 2008). Ressalta-se ainda que o adesivo ureia-formaldeído é polimerizado em meio ácido, fato que poderia ser dificultado pelo tratamento com $\mathrm{NaOH}$ e, assim, interferir na qualidade de colagem (Pizzi, 1994). 
As modificações que ocorreram nas partículas submetidas aos tratamentos, quando expostas à umidade, tornam-se um fator limitante na utilização da matéria prima (Machado et al., 2017). Algumas pesquisas reportadas na literatura abordaram o mesmo comportamento (Barbirato et al., 2014; Machado et al., 2017; Carvalho et al., 2015) e os autores relacionaram o aumento das propriedades físicas com o número de partículas de resíduos que são utilizadas, que geralmente possuem baixa densidade, prejudicando a estabilidade dimensional.

A NBR 14810 (ABNT, 2013) não estipula valores para o IE2h, apenas para o IE24h e permite uma porcentagem máxima de $18 \%$. Dessa forma, nenhum dos painéis atendeu ao mínimo especificado pela norma, portanto, os tratamentos foram ineficazes em relação às melhorias na estabilidade dimensional dos painéis e por isso não são indicados para serem utilizados em locais de uso interno em condições secas (Tipo 2).

\subsection{Propriedades mecânicas dos painéis}

Na Tabela 3 estão descritos os valores médios para as propriedades mecânicas testadas.

Tabela 3. Valores médios das propriedades mecânicas dos painéis aglomerados.

\begin{tabular}{ccccccc}
\hline Tratamentos & $\begin{array}{c}\text { MOR } \\
(\mathrm{MPa})\end{array}$ & $\begin{array}{c}\text { MOE } \\
(\mathrm{MPa})\end{array}$ & $\begin{array}{c}\text { TP } \\
(\mathrm{MPa})\end{array}$ & $\begin{array}{c}\text { DJ } \\
(\mathrm{MPa})\end{array}$ & $\begin{array}{c}\text { RAPS } \\
(\mathrm{N})\end{array}$ & $\begin{array}{c}\text { RAPT } \\
(\mathrm{N})\end{array}$ \\
\hline Controle & $6,14^{(2,79)} \mathrm{a}$ & $1.050^{(0,23)} \mathrm{a}$ & $0,41^{(0,19)} \mathrm{a}$ & $2,02^{(0,10)} \mathrm{a}$ & $180,40^{(11,58)} \mathrm{a}$ & $42,35^{(17,15)} \mathrm{a}$ \\
Água & $9,54^{(4,04)} \mathrm{a}$ & $2.020^{(0,27)} \mathrm{b}$ & $0,93^{(0,55)} \mathrm{c}$ & $2,12^{(0,14)} \mathrm{a}$ & $77,45^{(3,03)} \mathrm{a}$ & $66,15^{(25,95)} \mathrm{b}$ \\
$\mathrm{NaOH}$ & $8,34^{(3,59)} \mathrm{a}$ & $1.300^{(1,30)} \mathrm{a}$ & $0,63^{(0,31)} \mathrm{b}$ & $2,15^{(0,08)} \mathrm{a}$ & $81,25^{(13,28)} \mathrm{a}$ & $67,80^{(7,60)} \mathrm{b}$ \\
\hline
\end{tabular}

*Nota: Médias seguidas de mesma letra não diferem na coluna não diferem entre si (Skott-Knott; $\mathrm{p}>0.05$ ); Valores entre parênteses representam o desvio padrão. Fonte: Autores (2021).

Em relação ao módulo de ruptura (MOR) observa-se que os tratamentos aplicados nas partículas não promoveram diferenças significativas nesta propriedade mecânica. Porém, observa-se uma tendência de melhoria nesta característica, uma vez que houve um acréscimo nos valores da resistência dos painéis produzidos com partículas tratadas. Para o módulo de elasticidade (MOE), por outro lado, foram constatadas diferenças significativas entre os tratamentos. Os painéis produzidos com partículas controle e tratadas com $\mathrm{NaOH}$ evidenciaram médias iguais, mas diferiram daqueles constituídos com partículas tratadas em água, painéis que apresentaram um acréscimo significativo na resistência.

Em parte, tal resultado pode ser explicado devido a maior remoção dos extrativos com água (Tabela 1), que melhorou a qualidade de colagem partícula-partícula, reduzindo os espaços vazios no painel, e, desse modo, no momento de aplicação da força, os painéis apresentaram maior resistência. Outro fator que pode ter influenciado neste aspecto é a maior quantidade de partículas necessárias para a formação do colchão, uma vez que, o tratamento com água removeu maiores quantidades de extrativos dos lumes e espaços celulares (Tabela 1), e, por isso, foi necessário um maior número de partículas para formar o colchão, resultando em uma estrutura mais compacta e mais resistente.

Os valores do MOR e do MOE foram superiores aos obtidos por Martins et al. (2018) que relataram valores médios de 4,16 MPa para o MOR e 627,23 MPa para o MOE. Veloso et al. (2020) produziram painéis aglomerados com partículas de pecíolo de jupati associadas com partículas de madeira de eucalipto $(0,10,20$ e $30 \%)$ encoladas com teor de $9 \%$ de adesivo a base de ureia-formaldeído; a densidade nominal encontrada neste trabalho foi de $0,60 \mathrm{~g} / \mathrm{cm}^{3}$ e os parâmetros de prensagem foram: temperatura de $140{ }^{\circ} \mathrm{C}$ e pressão de $4,0 \mathrm{MPa}$, durante 10 minutos. Para a proporção de $30 \%$ de resíduo e $70 \%$ de madeira os autores obtiveram valores médio de 4,75 MPa para o MOR e 1.084,57 MPa para o MOE. 
Guimarães et al. (2019) observaram uma tendência de redução do MOR e do MOE com a adição de casquilho de soja na composição dos painéis. Outros pesquisadores que trabalharam com resíduos e observaram a mesma situação, tais como: Cravo et al. (2015), Guimaraes Júnior et al. (2016), Souza et al. (2017), Vieira et al. (2020), Brito et al. (2021), dentre outros.

A NBR 14810 (ABNT, 2013) estipula valores mínimos para o MOR e MOE, 11 MPa e 1.600 MPa, respectivamente. Com base nos dados da Tabela 4, verifica-se que os painéis do tratamento T3 não atingiram as especificações para o MOR, por uma diferença mínima na resistência equivalente a 13,28\%; porém, os painéis do referido tratamento estão em conformidade com o valor mínimo requerido pela norma para o MOE.

Para tração perpendicular (Tabela 4), observa-se que todos os tratamentos diferiram entre si e os painéis constituídos com partículas tratadas com $\mathrm{NaOH}$ evidenciaram um incremento na qualidade de colagem, equivalente a $35 \%$ em relação aos painéis produzidos com partículas controle e $56 \%$, para aqueles fabricados com partículas tratadas com água fria. Conforme comentado anteriormente os resultados obtidos podem ter sido influenciados pelo maior percentual de extrativos removidos das partículas tratadas com água (Tabela 1), que aumentou a adesão, evidenciando melhor qualidade de colagem. Além disso, conforme comentado anteriormente, pode ter ocorrido uma baixa polimerização do adesivo UF com o tratamento alcalino das partículas e, por isso a TP foi menos eficiente que os painéis produzidos com partículas tratadas em água fria. O resultado foi superior ao obtido por Martins et al. (2018) que encontraram 0,19 MPa para a tração perpendicular de painéis constituídos com a mesma proporção de partículas utilizada nesta pesquisa e Guimarães et al. (2019) que reportaram valor médio de 0,20 MPa. A NBR 14810 (ABNT, 2013) estipula o valor mínimo de 0,35 MPa para tração perpendicular e, dessa forma, todos os painéis atenderam tal exigência.

Para a dureza Janka (DJ) (Tabela 3) não foram constatadas melhorias com os tratamentos aplicados nas partículas pois todos os tratamentos apresentaram a mesma média. Baldin et al. (2016) produziram painéis aglomerados com Eragrostis plana (capim-annoni) e madeira de Pinus sp., em três proporções. A resina utilizada foi a base de UF com $9 \%$ de teor de sólidos e densidade nominal de $0,70 \mathrm{~g} / \mathrm{cm}^{3}$. Os parâmetros de prensagem foram: pressão de $30 \mathrm{kgf} / \mathrm{cm}^{2}$; temperatura de $180{ }^{\circ} \mathrm{C}$ e tempo de prensagem de 10 minutos. Para os painéis fabricados com proporção de partículas de 50:50 de cada material lignocelulósico foi obtido valor médio de 14,87 MPa, superior ao obtido no presente estudo. A NBR 14810 (ABNT, 2013) estabelece 1,0 MPa como valor mínimo exigido para o tipo de painel, em questão. Assim, todos os painéis atenderam a exigência da norma.

Para o arrancamento de parafusos de superfície (APS) não foram constatadas melhorias com os tratamentos das partículas. Em relação ao arrancamento de parafuso de topo (APT) observou-se um ganho na resistência para os painéis produzidos com partículas tratadas em água e hidróxido de sódio. Este comportamento é condizente com a propriedade de tração perpendicular. Conforme foi observado, a resistência a TP aumentou significativamente com o tratamento das partículas, que pode ter refletido neste ensaio de APT, considerando que este teste é feito a partir da inserção do parafuso no centro do painel. Quanto maior a qualidade de colagem, maior será a resistência necessária para o arrancamento do parafuso da camada central do painel. Portanto, pode-se afirmar que ambos os tratamentos retiraram extrativos de forma significativa para a melhoria do APT, além da maior compactação das partículas na estrutura interna do painel.

Brito et al. (2020) fabricaram painéis aglomerados com resíduos de bagaço de cana de açúcar e partículas de bambu em diferentes proporções (100:0, 75:25, 50:50, $25: 75$ e 0:100). Adotou-se a densidade nominal de $0,65 \mathrm{~g} / \mathrm{cm}^{3}, 10 \%$ de resina ureia formaldeído, $1 \%$ de parafina e os parâmetros do ciclo de prensagem foram: pressão de $35 \mathrm{kgf} / \mathrm{cm}^{2}$, temperatura de $180{ }^{\circ} \mathrm{C}$ durante 10 minutos. Para a proporção de 50:50 os autores obtiveram valores médio de 739,00 e 545,00 N, para o APS e APT, respectivamente, superiores aos obtidos nesta pesquisa.

Vieira et al. (2020) produziram painéis aglomerados com resíduos de cevada industrial e partículas de Pinus spp. em diferentes proporções (100:0, 75:25, 50:50, $25: 75$ e 0:100). Adotou-se a densidade nominal de $0,65 \mathrm{~g} / \mathrm{cm}^{3}, 12 \%$ de resina ureia formaldeído, $1 \%$ de parafina e os parâmetros do ciclo de prensagem foram: pressão de $40 \mathrm{kgf} / \mathrm{cm}^{2}$ e temperatura de $160{ }^{\circ} \mathrm{C}$ 
durante 8 minutos. Para a proporção de 50:50 os autores obtiveram valores médios de 929,17 e 707,00 N, para o APS e APT, respectivamente, superiores aos obtidos nesta pesquisa.

A NBR 14810 (ABNT, 2013) não estipula valor padrão para essas variáveis, porém considerando como referência os requisitos da antiga NBR 14810 (2006), que estipulava valores mínimos de resistência ao arranque de parafusos de $1.020 \mathrm{~N}$ (superfície) e de $800 \mathrm{~N}$ (topo), verifica-se que nenhum dos tratamentos avaliados atenderia essa exigência.

\section{Conclusão}

Os tratamentos das partículas de eucalipto e resíduo de soja, com água e $\mathrm{NaOH}$, não foram viáveis para melhorar a estabilidade dimensional dos painéis. O tratamento em água foi equivalente ao resultado obtido para as partículas controle, sendo assim para evitar gastos, não seria indicado a utilização destes tratamentos, a menos que as variáveis de produção sejam modificadas.

Para as propriedades mecânicas, os painéis produzidos com partículas tratadas com água atenderam as exigências para o MOE. Para as propriedades de tração perpendicular e dureza Janka, os valores também foram atendidos, conforme as designações da NBR 14810 (ABNT, 2013). As outras propriedades como o MOR, APS e APT não atenderam aos valores mínimos especificados pela norma.

Os tratamentos aplicados nas partículas de soja e eucalipto não demonstraram eficiência para as propriedades físicas, porém algumas propriedades mecânicas atenderam aos requisitos mínimos da NBR 14810 (2013), para painéis não estruturais para uso interno em ambiente seco (Tipo P2).

Diante destes resultados preliminares positivos, principalmente através do tratamento com $\mathrm{NaOH}$, novos estudos complementares são requeridos para uma maior caracterização dos painéis com pré-tratamento de partículas e, mediante ao atendimento aos padrões normativos, aplicação sustentável destes a larga escala. Dessa forma, a pesquisa abre espaços para diversas pesquisas inovadoras, com temáticas abrangendo soluções para a utilização dos resíduos gerados e seu reaproveitamento, levando em consideração as questões econômicas e ambientais.

\section{Agradecimentos}

Os autores agradecem o apoio financeiro das agências de fomento à pesquisa, Coordenação de Aperfeiçoamento de Pessoal de Nível Superior (CAPES), Conselho Nacional de Desenvolvimento Científico (CNPQ) e Fundação de Amparo à Pesquisa do Estado de Minas Gerais (FAPEMIG).

\section{Referências}

Associação Brasileira De Normas Técnicas - ABNT (2013). NBR 14810: Painéis de partículas de média densidade. Rio de Janeiro.

Associação Brasileira de Normas Técnicas - ABNT (2006). NBR 14810-3: Chapas de madeira aglomerada: métodos de ensaios. Rio de Janeiro.

Associação Brasileira de Normas Técnicas - ABNT (2010). NBR 14853: Madeira: determinação do material solúvel em etanol-tolueno e em diclorometano e em acetona. Rio de Janeiro.

Associação Brasileira de Normas Técnicas - ABNT (2010). NBR 7989: Pasta celulósica e madeira: determinação de lignina insolúvel em ácido. Rio de Janeiro.

Baldin, T., Silveira, A. G., Vidrano, B. R. A., Cancian, L. C., Spatt, L. L. \& Haselein, C. R. (2016). Qualidade de painéis aglomerados produzidos com diferentes proporções de madeira e capim-annoni. Revista Brasileira de Ciencias Agrárias, 11(3), 230-237. https://doi.org/10.5039/agraria.v11i3a5376

Barbirato, G., Fiorelli, J., Barrero, N. G., Pallone, E. M. de J. A., Lahr, F. A. R., Cristoforo, A. L. \& Savastano Júnior, H. (2014). Painel aglomerado híbrido de casca de amendoim reforçado com partículas de madeira itaúba. Ciência Florestal, 24(3), 685-697. http://doi.org/10.5902/1980509815726.

Bazzetto, J. T. L., Bortoletto Junior, G. \& Brito F. M. S. (2019). Effect of particle size on bamboo particle board properties. Floresta e Ambiente, 26(2). https://doi.org/10.1590/2179-8087.012517. 
Brito, F. M. S., Bortoletto Junior, G., Paes, J. B., Belini, U. L. \& Tomazello-Filho, M. (2020). Technological characterization of particleboards made with sugarcane bagasse and bamboo culm particles. Construction and Building Materials, 262, e120501. https://doi.org/10.1016/j.conbuildmat.2020.120501

Brito, F. M. S., Silva, P. X. S., Palumbo, S. K. C., Guimarães Júnior, J. B. \& Mendes, L. M. (2021). Technological characterization of particleboards constituted with pistachio shell (Pistacia vera) and Pinus oocarpa wood. Revista Brasileira de Ciências Agrárias, 16(2), e8902. https://doi.org/10.5039/agraria.v16i2a8902

Brito, F. M. S., Bortoletto Júnior, G. (2019). Thermal modification of sugarcane waste and bamboo particles for the manufacture of particleboards. Revista Árvore, 43(1). https://doi.org/10.1590/1806-90882019000100012.

Brito, F. M. S. \& Bortoletto Júnior, G. (2020). Properties of particleboards manufactured from bamboo (Dendrocalamus asper). Revista Brasileira De Ciências Agrárias, 15, 1-10. https://doi.org/10.5039/agraria.v15i1a7245

Cao, Y., Song, W. Yang, Z. Chen, Z. \& Zhang, S. (2017). The Properties of Particleboard Made from Alkalinetreated Wheat Straw and Methylene Diphenyl Diisocyanate Binder. BioResources, 12(2): 3265-3276

Carvalho, A. G., Andrade, B. G. de., Cabral, C. P. T. \& Vital, B. R. (2015). Efeito da adição de resíduos de poda da erva-mate em painéis aglomerados. Revista Árvore 39(1): 209-214. https://doi.org/10.1590/0100-67622015000100020.

César, A. A. S., Bufalino, L., Mendes, L. M., Mesquita, R. G.A., Protásio, T. P., Mendes, R. F. \& Andrade, L. M. F. (2017). Transforming rice husk into a high-added value product: Potential for particleboard production. Ciência Florestal, 27(1), 303-313. https://doi.org/10.5902/1980509826468

Companhia Nacional De Abastecimento - CONAB (2020). Acompanhamento da safra brasileira: Grãos - Safra 2019/20 - Décimo Levantamento, 7(10), Brasília.

Cravo, J. C. M., Sartori, D. de L., Fiorelli, J., Balieiro, J. C. de C. \& Savastano Júnior, H. (2015). Painel aglomerado de resíduos agroindustriais. Ciência Florestal, 25 (3), 721-730. https://doi.org/10.5902/1980509819675.

Empresa brasileira de pesquisa e agropecuária - EMBRAPA (2019). Transferência de tecnologia florestal. Colombo.

Garcia, H. V. S. G., Furtini, A. C. C., Brito, F. M. S., Santos, C. A., Ribeiro, D. A., Guimarães Júnior, J. B \& Mendes, L. M. (2021). Desempenho de painéis de madeira laminada colada cruzada. Constituídos com eucalipto, seringueira e bambu. Research, Society and Development, 10(8), e33210817181. http://doi.org/10.33448/rsd-v10i8.17181

Gonçalves, F. G., Segundinho, P. G. A., Fassarella, M. V., Minini, D., Tinti, V. P., Lopez,Y, M. \& Felberg, M. J. K. F. S. (2021). Relação entre propriedades elásticas e biológicas em painéis aglomerados feitos com resíduos de madeira de eucalipto. Revista Matéria, 26(3). https://doi.org/10.1590/S1517707620210003.13048

Guimarães Júnior, J. B., Xavier, M. M., Santos, T. S., Protásio, T. P., Mendes, R. F., Mendes. L. M. (2016). Inclusão de resíduo da cultura de sorgo em painéis aglomerados de eucalipto. Pesquisa Florestal Brasileira, 36(88), 435-442. https://doi.org/10.4336/2016.pfb.36.88.1036.

Guimarães, B. M. R., Mendes, L. M., Tonoli, G. H. D., Bufalino, L., Mendes, R. F. \& Guimarães Junior, J. (2014). Chemical treatment of banana tree pseudostem particles aiming the production of particleboards. Science and Agrotechnology, 38(1), 43-49. https://doi.org/10.1590/S1413-70542014000100005.

Guimarães, I. L., Veloso, M. C. R. A., Lisboa, F. J. N., Mendes, R. F., Mendes, L. M., Farrapo, C. L. \& Guimarães Junior, J. B. (2019). Aproveitamento do casquilho de soja para a produção de painéis aglomerados convencionais de baixa densidade. Revista Brasileira de Ciências Agrárias, 14(2), e5643. https://doi.org/10.5039/agraria.v14i2a5643

Instituto Brasileiro de Atuária - IBA (2021). Relatório anual.

Iwakiri, S. \& Trianoski, R. (2020). Painéis de madeira reconstituída. Universidade Federal do Paraná.

Iwakiri, S. (2005). Painéis de madeira reconstituída. Universidade Federal do Paraná

John, M. J. \& Anandjiwala, A. D. (2008). Recent developments in chemical modification and characterization of natural fiber-reinforced composites. Polymer Composites, 29(2), 187-207. https://doi.org/10.1002/pc.20461.

Joseleau, J. P., Imai, T., Kuroda, K. \& Ruel, K. (2004). Detection in situ and characterization of lignin in the G-layer of tension wood fibres of Populus deltoids. Planta, 219(2), 338-345. https://doi.org/10.1007/s00425-004-1226-5.

Jumhuri, N., Hashim, R. Sulaiman, O., Nadhari, W. N. A. W., Salleh, K. M., Khalid, I., Saharudin, N. I. \& Razali, M. Z. (2014). Effect of treated particles on the properties of particleboard made from oil palm trunk. Materials and Design, 64, 769-774. https://doi.org/10.1016/j.matdes.2014.08.053

Khedari, J., Nankongnab, N., Hirunlabh, J. \& Teekasap, S. (2004). New low-cost insulation particleboards from mixture of durian peel and coconut coir. Building and Environment, 39(1), 59-65. https://doi.org/10.1016/j.buildenv.2003.08.001.

Kin, Z. (1980). Hemicelulozy: chemia i wykorzystanie. Państwowe Wydawnictwo Rolnicze i Leśne, Varsóvia: WYDAWCA: PWRiL - Państwowe Wydawnictwo Rolnicze i Leśne, 232p.

Klock, U. \& Andrade, A. S. (2013). Química da madeira. Universidade Federal do Paraná.

Machado, N. A. F., Furtado, M. B., Parra-Serrano, L. J., Parente, M. de O. M., Fiorelli, J. \& Savastano Júnior, H. (2017). Painéis aglomerados fabricados com resíduos do coco babaçu. Revista Brasileira de Ciências Agrárias, 12(2), 202-209. https://doi.org/10.5039/agraria.v12i2a5434

Martins, E. H., Vilela, A. P., Mendes, R. F., Mendes, L. M., Vaz, L. E. V. S. B. \& Guimarães Junior, J. B. (2018). Soybean waste in particleboard production. Science and Agrotechnology ,42(2), 186-194. http://dx.doi.org/10.1590/1413-70542018422015817. 
Research, Society and Development, v. 11, n. 2, e29511225762, 2022

(CC BY 4.0) | ISSN 2525-3409 | DOI: http://dx.doi.org/10.33448/rsd-v11i2.25762

Matos, A. T. (2005). Curso sobre tratamento de resíduos agroindustriais. $35 \mathrm{p}$.

Moraes, A. G. O. (2010). Estudo do íon boroidreto como agente protetor de fibras de sisal no tratamento em meio alcalino. 2010.81 p. Dissertação (Mestrado em Ciência e Tecnologia dos Materiais) - Universidade Federal do Rio Grande do Sul, Porto Alegre.

Pizzi, A. (1994). Advanced wood adhesives technology. M. Dekker, 289p.

Scatolino, M. V., Costa, A. O., Guimarães, J. B., Protásio, T. P, Mendes, R. F. \& Mendes, L. M. (2017). Eucalyptus wood and coffee parchment for particleboard production: Physical and mechanical properties. Science and Agrotechnology, 41(2), 139-146. http://dx.doi.org/10.1590/141370542017412038616 .

Sellers, T., Mcsween Junior, J. R. \& Nearn, W. T. (1988). Gluing of Eastern hardwoods: a review. New Orleans. https://doi.org/10.2737/SO-GTR-71.

Sette Júnior, I, C. R., Deus Júnior, J. C., Tomazello Filho, M., Pádua, F. A., Calil, F. N. \& Laclau, J. P. (2014). Alterações na qualidade da madeira de Eucalyptus grandis causadas pela adubação mineral. Cerne, 20(2). https://doi.org/10.1590/01047760.201420021499.

Silva, D. W., Farrapo, C. L. Ribeiro, D. P., Mendes, R. F., Mendes, L. M., Scolforo, J. R. S. (2015). MDP com partículas de eucalipto e palha de milho. Scientia Forestalis, 43(108), 853-862. http://dx.doi.org/10.18671/scifor.v43n108.10.

Souza, J. T., Menezes, W. M., Haselein, C. R., Baldin, T., Azambuja, R. R. \& Morais, W. W. C. (2017). Avaliação das propriedades físico-mecânicas de painéis de casca de arroz e adesivo tanino-formaldeído. Ciência Florestal, 27(3), 1003-1015. https://doi.org/10.5902/1980509828674

Surdi, P. G., Bortoletto Júnior, G., Castro, V. R., Brito, F. M. S., Berger, M. S. \& Zanuncio, J. C. (2019). Particleboard production with residues from mechanical processing of Amazonian woods. Revista Árvore, 43(1). http://doi.org/10.1590/1806-90882019000100002

Sweet, M. S. \& Winandy, J. E. (1999). Influence of Degree of Polymerization of Cellulose and Hemicellulose on Strength Loss in Fire-Retardant-Treated Southern Pine. Holzforschung, 53(3), 311-317. https://doi.org/10.1515/HF.1999.051.

Veloso, M. C. R. A., Lopes, F. M., Furtini, A. C. C., Silva, M. G., Mendes, L. M. \& Guimarães Júnior, J. B. (2020). Low-density particleboard properties produced with jupati particles and eucalyptus wood. Revista Brasileira de Ciências Agrárias, 15(4), e8414. https://www.doi.org/10.5039/agraria.v15i4a8414.

Vieira, H. C., D`Angelo Rios, P., Cunha, A. B., Kniess, D. C., Gaa, A. Z. N., Ricardo, G. C. \& Balduino Junior, A. L. (2020). Resíduo de cevada industrial para a produção de painéis aglomerados. Revista de Ciências Agroveterinárias, 19(3), 365-372. https://doi.org/10.5965/223811711932020365

Zanuncio, A. J. V., Nobre, J. R. C., Motta, J. P. \& Trugilho, P. F. (2014). Química e colorimetria da madeira de Eucalyptus grandis W. Mill ex Maiden termorretificada. Revista Árvore, 38(4). https://doi.org/10.1590/S010067622014000400020.

Zhang, L. \& Hu, Y. (2014). Novel lignocellulosic hybrid particle board composites made from rice straws and coir fibers. Materials and Design, 55, 19-26. https://doi.org/10.1016/j.matdes.2013.09.066

Zheng, Y., Pan, Z., Zhang, R., Jenkins, B. M. \& Blunk, S. (2005). Medium-density Particleboard from Saline Jose Tall Wheatgrass. American Society of Agricultural and Biological Engineers. https://doi.org/10.13031/2013.19578. 